\title{
Desquamative esophagitis due to pemphigus vulgaris
}

A 48-year-old woman was admitted to our hospital with hematemesis that occurred after dinner. Two years previously, she had been diagnosed as having pemphigus vulgaris on the basis of bullous lesions in the oral mucosa and the presence of anti-desmoglein 3 (Dsg3) antibodies. Esophagogastroduodenoscopy (EGD) revealed desquamation of the mucosa, blood oozing from the entire esophagus ( $\bullet$ Fig. 1), and a whitish strip, which was exfoliated esophageal mucosa, hanging loosely from the cardia ( Fig. 2). Prednisolone (30 mg/day) was administered. Two weeks later, EGD showed a dramatic improvement in the esophageal mucosa, which had an almost normal appearance (๑ Fig. 3).

Pemphigus vulgaris is a rare, autoantibody-mediated, blistering disease of the skin and mucosal membrane. The most frequently involved mucosa is the oral mucosa; oral mucosal lesions occur in nearly all patients during the course of the disease. It was long considered that the esophageal mucosa is rarely involved in pemphigus vulgaris [1]. However, in 1999, Gomi et al. reported that patients with pemphigus vulgaris had a high frequency (87.5\%) of esophageal lesions (bullae or exfoliated erosions of bullae); these lesions led to exfoliative esophagitis [2]. Patients with mucosal pemphigus vulgaris have autoantibodies against the desmosomal cadherin Dsg3. These autoantibodies are responsible for loss of cohesion between epidermal cells, resulting in blister formation. Such a high frequency of esophageal involvement may be because Dsg3 is highly expressed in the stratified squamous epithelium of the esophagus as well as that of the oral mucosa [3]. Nikolsky's sign, a well-described clinical sign of pemphigus vulgaris, is positive when manual pressure on the skin of patients elicits the separation of the epidermis. A positive Nikolsky's sign has also been reported on the mucosal membrane of the esophagus and uterine cervix in patients with pemphigus vulgaris [4,5]. In conclusion, when clinicians encounter idiopathic esophageal lesions and extensive bleeding from the esophagus, especially with oral mucosal lesions, pemphigus vulgaris should be considered in the differential diagnosis.

\section{Competing interests: None}

Endoscopy_UCTN_Code_CCL_1AB_2AC_3AH

Y. Matunaga ${ }^{1}$, A. Goto ${ }^{1}$, K. Fujii ${ }^{1}$,

H. Nakamura ${ }^{2}$, M. Itoh ${ }^{1}$, H. Wakasugi ${ }^{1}$,

K. Suzuki ${ }^{1}$, K. Yonezawa ${ }^{1}$, T. Abe ${ }^{1}$,

Y. Shinomura ${ }^{3}$

1 Department of Gastroenterology, Kushiro City General Hospital, Japan

2 Department of Dermatology, Kushiro City General Hospital, Japan

3 1st Department of Internal Medicine, Sapporo Medical University, Japan

\section{References}

1 Gellis S, Glass FA. Pemphigus: a survey of 170 patients admitted to Bellevue Hospital from 1911 to 1941. Arch Dermatol Syph 1941; 44: 321-326

2 Gomi H, Akiyama M, Yakabi Ket al. Oesophageal involvement in pemphigus vulgaris. Lancet 1999; 354: 1794

3 Shirakata Y, Amagai M, Hanakawa $Y$ et al. Lack of mucosal involvement in pemphigus foliaceus may be due to low expression of desmoglein 1. J Invest Dermatol 1998; 110: $76-78$

4 Coelho LK, Troncon LE, Roselino AM et al. Esophageal Nikolsky's sign in pemphigus vulgaris. Endoscopy 1997; 29: S35

5 Sagher F, Bercovici B, Romem R. Nikolsky sign on cervix uteri in pemphigus. Br J Dermatol 1974; 90: 407-411

Bibliography

DOI $10.1055 / \mathrm{s}-0030-1255569$

Endoscopy 2010; 42: E285

(C) Georg Thieme Verlag KG Stuttgart. New York . ISSN 0013-726X

Corresponding author

\section{A. Goto, MD}

Department of Gastroenterology

Kushiro City General Hospital

1-12, Shunkodai

Kushiro City

085-0822, Japan

Fax: +81-0154-414080

goa@sapmed.ac.jp

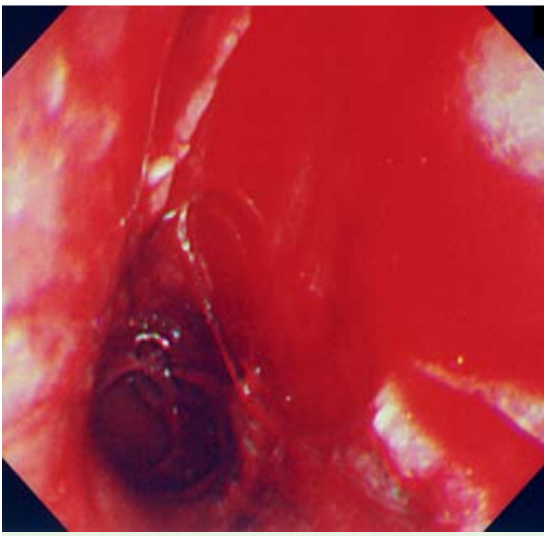

Fig. 1 Esophagogastroduodenoscopy (EGD) showing desquamation of the mucosa and blood oozing from the entire esophagus.

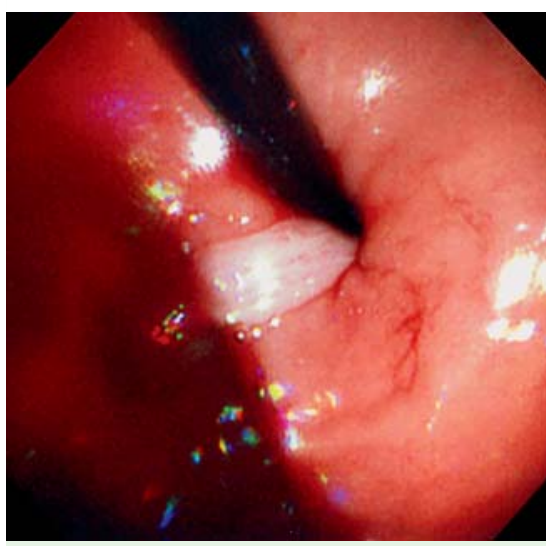

Fig. 2 EGD showing a whitish strip, which is exfoliated esophageal mucosa, hanging loosely from the cardia.

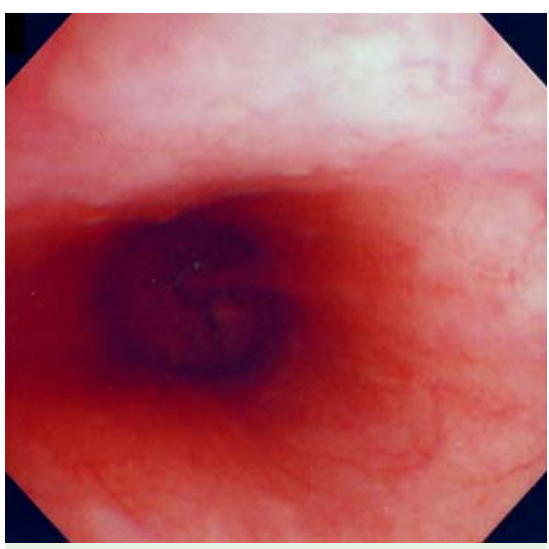

Fig. 3 EGD performed after 2 weeks, showing a dramatic improvement in the esophageal mucosa, which has an almost normal appearance. 